\title{
Hypertrichosis, CTCAE
}

National Cancer Institute

\section{Source}

National Cancer Institute. Hypertrichosis, CT CAE. NCI Thesaurus. Code C143561.

A disorder characterized by hair density or length beyond the accepted limits of normal in a particular body region, for a particular age or race. 